\title{
A Participatory Approach to Identify Key Areas for Sustainable Work Environment and Health in Employees with Flexible Work Arrangements
}

\author{
Sofie Bjärntoft ${ }^{1, *(\mathbb{D}}$, David M. Hallman ${ }^{1} \mathbb{(}$, Camilla Zetterberg ${ }^{1}$, Johan Larsson ${ }^{1,2,3}$, Johanna Edvinsson ${ }^{1}$ \\ and Helena Jahncke ${ }^{1}$ \\ 1 Department of Occupational Health Sciences and Psychology, University of Gävle, 80176 Gävle, Sweden; \\ david.hallman@hig.se (D.M.H.); camilla.zetterberg@hig.se (C.Z.); johan.larsson@lkab.com (J.L.); \\ johanna.edvinsson@hig.se (J.E.); helena.jahncke@afaforsakring.se (H.J.) \\ 2 Human Work Science, Luleå University of Technology, 97187 Luleå, Sweden \\ 3 Department of Occupational Health and Safety, LKAB, 98381 Gällivare, Sweden \\ * Correspondence: sofie.bjarntoft@hig.se
}

\section{check for}

updates

Citation: Bjärntoft, S.;

Hallman, D.M.; Zetterberg, C.;

Larsson, J.; Edvinsson, J.; Jahncke, H.

A Participatory Approach to Identify

Key Areas for Sustainable Work

Environment and Health in

Employees with Flexible Work

Arrangements. Sustainability 2021, 13,

13593. https://doi.org/

$10.3390 /$ su132413593

Academic Editor: Fred Zijlstra

Received: 16 November 2021

Accepted: 6 December 2021

Published: 9 December 2021

Publisher's Note: MDPI stays neutral with regard to jurisdictional claims in published maps and institutional affiliations.

Copyright: (c) 2021 by the authors. Licensee MDPI, Basel, Switzerland. This article is an open access article distributed under the terms and conditions of the Creative Commons Attribution (CC BY) license (https:/ / creativecommons.org/licenses/by/ $4.0 /)$.

\begin{abstract}
Flexible work arrangements are common worldwide, but knowledge on how to achieve a sustainable work environment is sparse. The aim of this study was to use a participatory approach to identify concrete suggestions and key areas for improvement that were considered relevant, effective, and feasible for promoting good work environment and health at organizational, work group and individual level (O-G-I), among office employees with flexible work arrangements. Eight focus group interviews (including 45 employees) were conducted in a large Swedish government agency in 2017. By using a Tree diagram approach, employees made a total of 279 suggestions for improvements, which were sorted into O-G-I levels and mapped into 18 key areas. We found that 13 key areas addressed organizational level (e.g., improving leadership, policy, job demands, and work efficiency), two key areas addressed group level (create common rules of availability and activity-based working), and three key areas addressed individual level (e.g., individuals' responsibility to clearly communicate their availability). The participatory process was effective in obtaining concrete suggestions and key areas in need of improvement, which may provide an action plan that can guide organizations in developing interventions to promote good work environment and health in flexible work.
\end{abstract}

Keywords: job autonomy; participative; work environment; health promotion; sustainable work

\section{Introduction}

The increase in digitalization and use of information and communication technology (ICT) enables organizations to offer employees more flexibility in the way they work. In Europe in 2019, approximately $40 \%$ of employees reported having some flexibility in when they worked, with the highest prevalence of flexibility reported in Sweden and Finland [1]. Before the pandemic, 65\% of the Swedish workforce could decide over when they worked (46\% with certain restrictions and 19\% could fully decide) [1], and around 35\% worked remotely to some extent [2]. Flexible work has becoming increasingly common due to the pandemic, mainly in terms of working from home as a means to reduce the spread of the virus [3]. Depending on organizational and individual needs, flexible work arrangements can present in different ways, both in terms of where and when to work (e.g., telework and flexibility in time) and how to perform the work (e.g., use of technical tools and activity-based offices) [4].

Both positive and negative effects have been reported for the effect of flexible work on working conditions and health $[4,5]$. Some reviews suggest employees with flexible work have improved job performance and organizational commitment [6], reduced somatic 
symptoms, better physical health [5], and improved mental health and psychological wellbeing [4]. These positive changes may result from increased work-time control (WTC) that facilitates a better combination between work and personal life $[4,6,7]$. WTC has previously been associated with beneficial outcomes in terms of reduced stress and strain, improved health [5], reduced work-life conflicts [6], and improved work-related wellbeing [4]. However, other studies indicate that flexible work is associated with reduced mental health [4]. Flexible work may result in a higher extent of employee accessibility to work in terms of both time and space [6,7], resulting in expectations of after-hours availability $[6,8]$. The lack of boundaries around work may adversely affect psychological detachment from work and in turn lead to work-family conflicts [7], which has previously been associated with work overload [6], and poor mental health [9]. Thus, previous findings also raise warnings and call for interventions to reduce health risks and to strengthen the benefits of flexible work to improve work environment and employee health.

Developing health promoting and sustainable work has been identified as a challenge in occupational health science [10]. Studies on the development and/or evaluation of interventions aimed at improving working conditions and health in flexible work are sparse. In recent years, interventions have mainly addressed an organizational level by flexible work programs or work-family policies, but still, organizational- and group interventions are least researched [11]. Interventions on an organizational level can focus on organizational structure, management styles and organizational culture and norms [12]. At a group level, interventions often address relationships and settings related to the work group, workplace, and work environment. On the individual level, interventions can target individual strategies to improve engagement at work, well-being, and work-life balance [12].

Several reviews $[11,13]$ call for a broader perspective and implementation of strategies targeting more than one level at the workplace. This perspective may be more effective in developing sustainable working conditions since the interaction between the levels often have stronger effect than interventions at one specific level [12]. This strategy can be seen as a part of System Theory approaches, which consists of different domains or levels that in some way affect each other [12]. Through this approach, it is possible to acquire a more holistic perspective of the key areas and organizational, group and individual levels (O-G-I) that require targeting for sustainable and health-promoting work environment interventions in flexible work. The approach of addressing multiple levels will be used in the present study.

For successful workplace interventions, a participatory approach which includes employees in the design stage of the intervention has been recommended [14]. One way of doing this is by using Participative Ergonomics (PE), that is; “The involvement of people in planning and controlling a significant amount of their own work activities, with sufficient knowledge and power to influence both processes and outcomes in order to achieve desirable goals" [15] (p. 1071). PE has previously focused on improvements in the work process or in the physical work environment which have resulted in positive effects on sustainable behavioral changes [14]. In addition, PE has been associated with improved design effectiveness for development work, ownership of change among the employees, and increased employee safety and job satisfaction [14]. However, according to our knowledge, no previous study has used PE to identify concrete suggestions for improvement to systematically identify key areas within different levels (O-G-I) at the organization. Such information would be useful for developing and implementing future interventions that could lead to evidence-based recommendations and guidelines for organizations offering flexibility at work. The aim of this study was to use a participatory approach to identify concrete suggestions and key areas for improvement that were considered relevant, effective, and feasible for promoting good work environment and health at organizational, work group and individual level (O-G-I), among office employees with flexible work arrangements. 


\section{Materials and Methods}

This study uses a mixed-methods design and is a part of a comprehensive research project on flexible work at a large governmental agency in Sweden (The Swedish transport administration). The overall project consists of (i) a work environment and health survey targeting all employees with flexible work arrangements (conducted in 2016), (ii) focus group interviews to identify concrete suggestions and key areas for improvement based on the results of the survey, and (iii) developing and implementing an intervention in a later phase. This study focusses on step two. Focus group interviews were conducted in October and November 2017, in which employees identified main themes, sub themes and concrete suggestions for developing a sustainable work environment and health in flexible work. Subsequent analyses divided the concrete suggestions by O-G-I level (organizational, work group and individual) and similar suggestions were mapped into key areas for improvement within each level. The present study has been approved by the Regional Ethical Review Board in Uppsala (2016/425/1). All participants provided their written informed consent.

\subsection{Participants}

We contacted the Human Resources department at the agency to assist with recruitment of participants to the study. The inclusion criteria were flexible work arrangement (i.e., flextime or non-regulated working hours) and the exclusion criteria was having a management position. Employees from eight departments in different cities were invited to participate. In total, 49 employees expressed interest in participating and were invited to join the group interview in their department, and 45 participants joined the interviews (three to eight participants per group). Descriptive statistics are shown in Table 1.

Table 1. Descriptive statistics for interview participants $(n=45)$ showing employment rate, work arrangement, sex, age, year of employment and level of education (number, percent and average).

\begin{tabular}{|c|c|c|c|c|}
\hline Variable & & Number & Percent & Average \\
\hline \multirow[t]{2}{*}{ Employment rate } & $100 \%$ (40 h/week) & 44 & $98 \%$ & \\
\hline & $<100 \%$ & 1 & $2 \%$ & \\
\hline \multirow[t]{3}{*}{ Work arrangement } & Non-regulated working hours & 37 & $82 \%$ & \\
\hline & Flextime & 7 & $16 \%$ & \\
\hline & Missing & 1 & $2 \%$ & \\
\hline \multirow[t]{2}{*}{ Sex } & Men & 23 & $51 \%$ & \\
\hline & Women & 22 & $49 \%$ & \\
\hline Age & & & & 44 years ( 26 to 59$)$ \\
\hline Years of employment & & & & 10 years $(0,5$ to 47$)$ \\
\hline \multirow[t]{3}{*}{ Level of education } & High school & 11 & $24 \%$ & \\
\hline & Vocational school & 5 & $11 \%$ & \\
\hline & University/college & 29 & $65 \%$ & \\
\hline
\end{tabular}

\subsection{Procedure and Data Collection}

The results of the work environment and health survey (2016) were reported to the organization, including departmental specific reports. The survey $(n=3259)$ examined risk- and promoting- factors in relation to several health-related outcomes in flexible work, including: stress, health, well-being, work-life balance and recovery (parts of the results have been published elsewhere [16]. The participants for the focus group interviews received the departmental specific report in connection with an invitation, and before the group interview started, the moderator gave a short presentation of the department's results, highlighting potential risk-and promoting-factors for a selection of health-related outcomes (e.g., health, recovery, and work-life balance). The presented results formed the basis for the discussions and concrete suggestions for developing a more sustainable work environment and improving health. Participants came from different cities around 
Sweden to one of the agency's offices for the approximately four-hour group interview. All interviews were recorded using dictaphones. Two researchers participated in each session, one as moderator and one as assistant. The researchers prepared with in-depth reading and methodological practice sessions. Prior the focus group interviews, the moderator informed the participants about the procedure, emphasizing that all participants should have their opinion heard, that it was not allowed to criticize ideas, and that the concrete suggestions made for improvements should be feasible. Subsequently, participants were asked to sign an informed consent to participate in the study.

A Tree diagram [17] was used to map responses to the question; "What interventions do you perceive as being most important to promote a good work environment and health in flexible work?". A Tree diagram is an established methodological tool, often used within quality improvement work to break down a question into suggestions for improvement $[17,18]$. The construction of a "top-down" diagram was used, including three levels, (main themes, sub themes and concrete suggestions), see Figure 1.

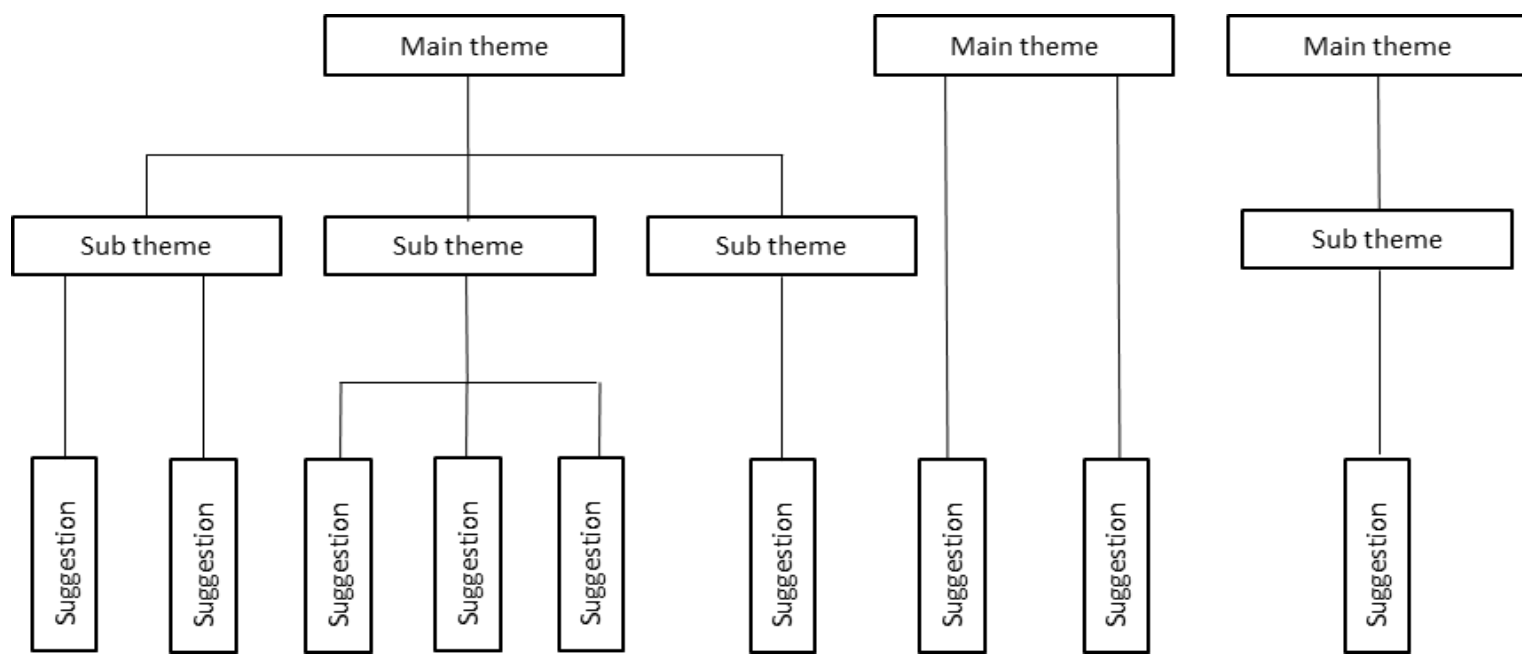

Figure 1. Example of a “top down” Tree diagram, inspired by Klefsjö, Eliasson and Kennerfalk's [17].

The moderator led the discussions, and the assistant took notes, recording themes, subthemes, and concrete suggestions on post-it notes. The assistant attached the post-it notes on a poster during the time they emerged in the discussion. The moderator only intervened if the discussions became irrelevant for the question, if any participant dominated the discussion or if the discussion ended in silence.

After constructing the Tree diagram, the group discussed all post-it notes on the poster and if anything was unclear to the researchers or participants, it was discussed. Lastly, each participant individually rated how effective and how feasible they perceived each of the concrete suggestions would be for promoting a good work environment and health in flexible work arrangements. The scale ranged from 0 (not at all) to 10 (to a high degree).

\subsection{Data Analysis}

Six researchers performed the analysis (see Figure 2). Four researchers worked together to reach consensus for all mapping, while two researchers reviewed and provided feedback after each analysis step. In the first step, each of the four researchers individually read all the suggestions from the focus group interviews and mapped each suggestion according to the O-G-I level (organizational, work group and individual) to which the suggestion applied. If researchers had mapped the suggestions differently, or if O-G-I level was not evident in the suggestion, the researchers discussed until consensus was reached. Some suggestions were potentially applicable to more than one level, in which case, all six researchers discussed until consensus was reached on which level was most applicable. 


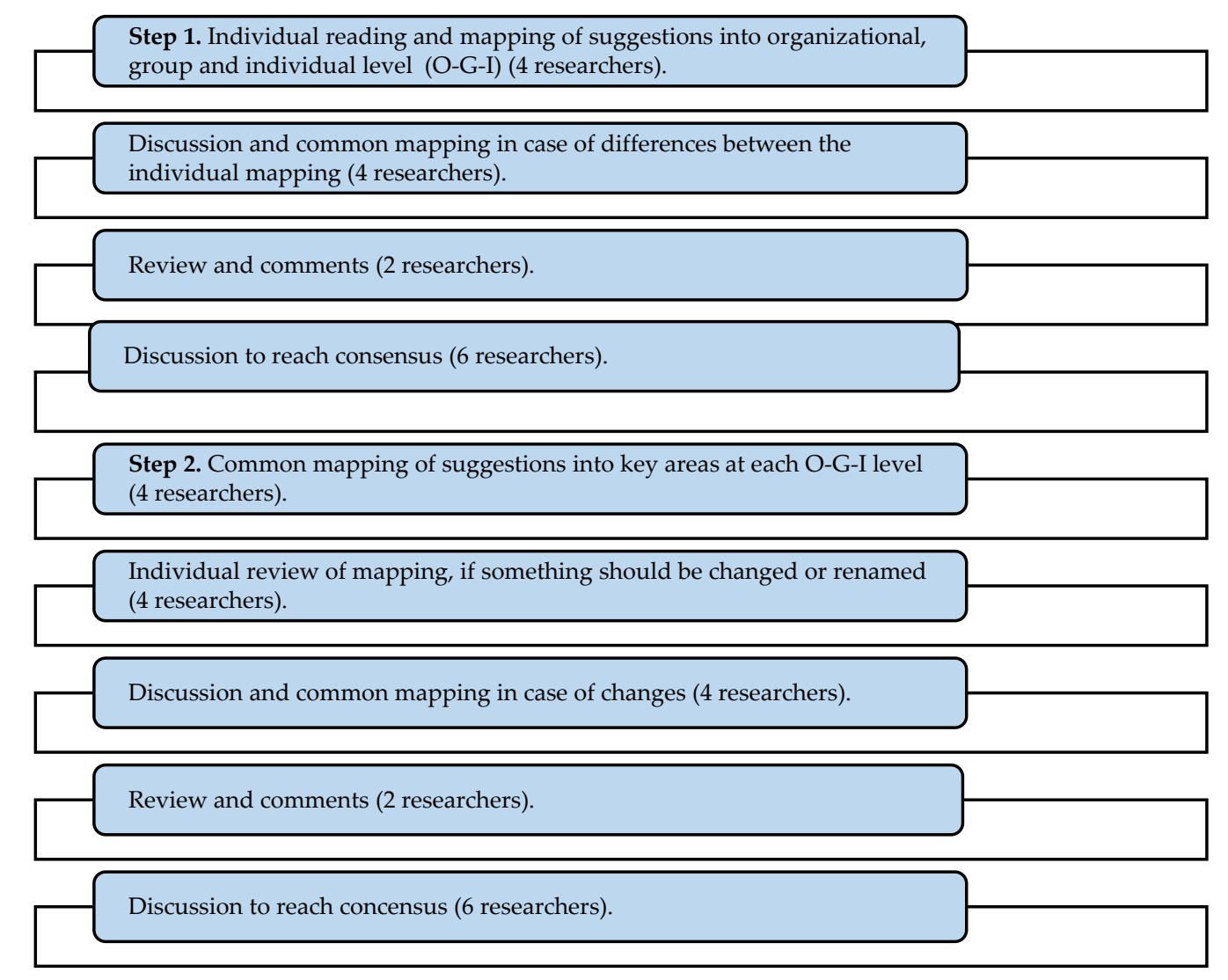

Figure 2. Analysis procedure: Step 1-mapping of each suggestion as organizational, group or individual level (O-G-I) action; Step 2-Thematic grouping of suggestions into key areas for each level.

In the second step, the four researchers evaluated the suggestions in each O-G-I level and mapped similar suggestions into key areas (and when necessary, sub-areas) for improvement. Each researcher then reviewed the key areas identified by the other researchers, and the key area names and designations were discussed and revised until consensus was reached. The ratings for effectiveness and feasibility (separately and combined) given by each participant were average for each sub-area, key area, and O-G-I level. The range of the mean value was also calculated for the combined effectiveness and feasibility score. Bubble diagrams were used to illustrate the key areas emerging for each O-G-I level (see Figures $3-5$ in the result section).

\section{Results}

Focus groups resulted in 279 concrete suggestions for improvements to promote good work environment and health in flexible work. Most suggestions were categorized as organizational level changes $(86 \%, \mathrm{n}=241)$, followed by the individual level $(8 \%, \mathrm{n}=21)$ and the group level $(6 \%, \mathrm{n}=17)$. In total, researchers identified 18 key areas, 13 at the organizational level, two at group level and three at the individual level. Employee ratings of effectiveness and feasibility were, on average, high for most key areas (range 6.8 to 8.5 ) and were similar across O-G-I levels: organizational (mean $=7.5$, range 6.8 to 8.5 ), group (mean $=7.7$, range 7.6 to 7.7), and individual level (mean $=7.4$, range 7.2 to 8.3). See Table 2 for a detailed description of ratings for all key areas and sub-areas within all O-G-I levels. Figures 3-5 summarize key areas by O-G-I level including the relative number of suggestions, effectiveness and feasibility score for each key area and number of groups contributing suggestions to the key area. 
Table 2. Description of identified key areas and sub areas promoting a good work environment and health in flexible work arrangements at organizational, group and individual level (O-G-I).

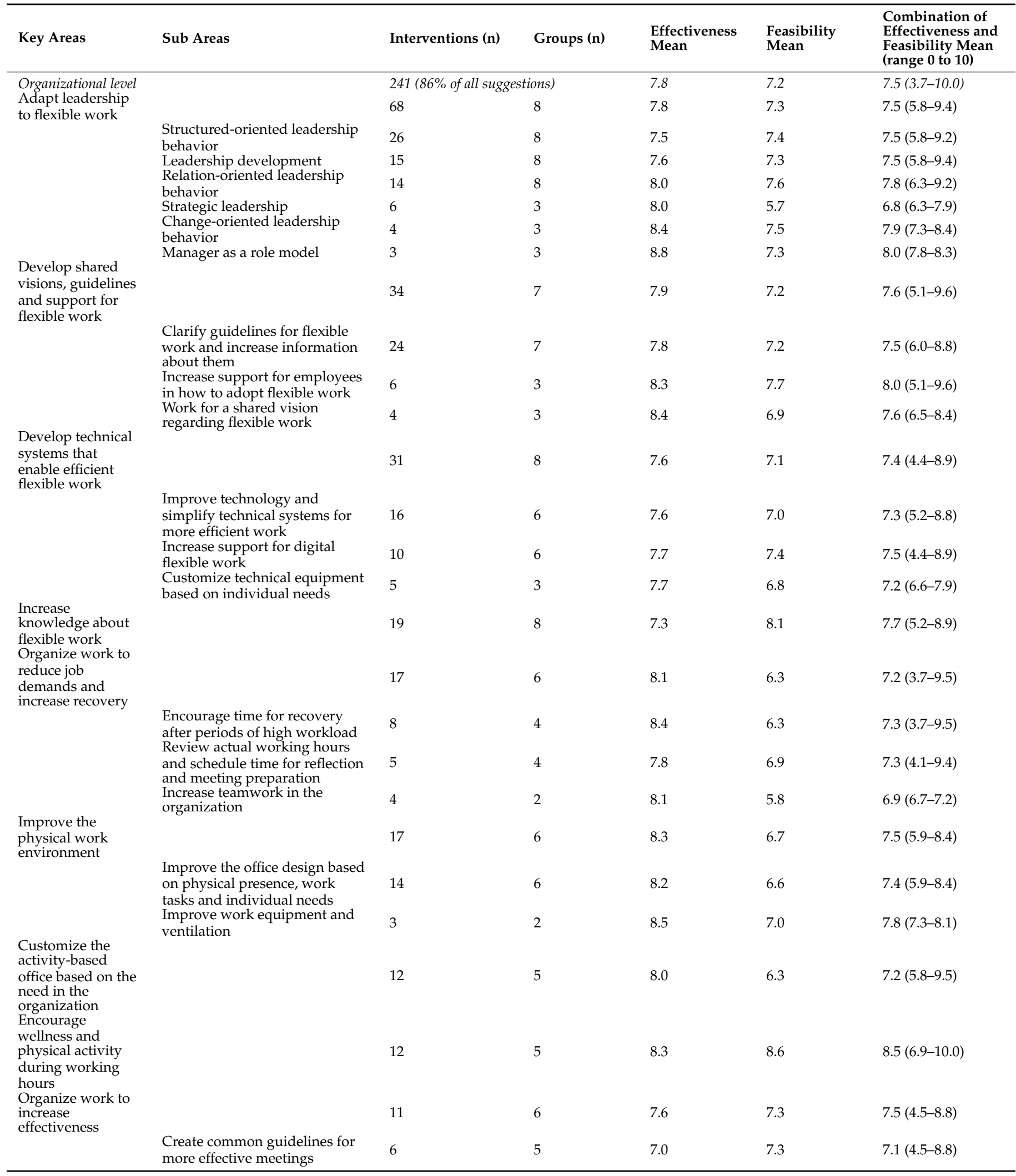


Table 2. Cont.

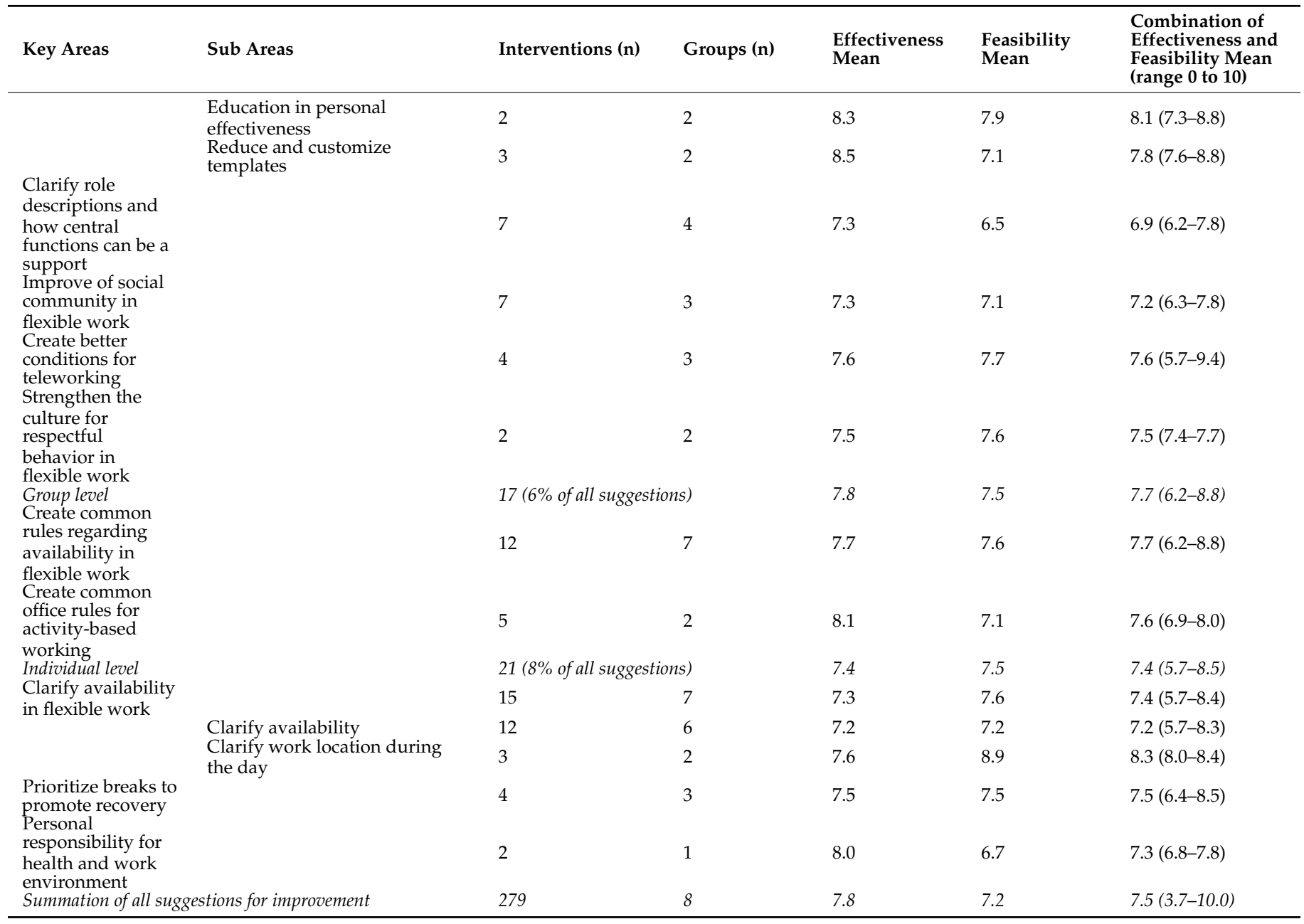

\subsection{Organizational Level}

On organizational level, the key area comprising the highest number of suggestions and contributions from all focus groups was leadership in flexible work (see Figure 3). This key area included five sub areas; structured oriented leadership behavior, leadership development, relation-oriented leadership behavior, strategic leadership, change-oriented leadership behavior and manager as a role model. Some examples of suggestions directed at the managerial level were to: increase knowledge about important leadership behaviors in flexible work; be a good role model by not sending emails outside of regular working hours; support employees in prioritizing work tasks and setting boundaries between work and personal life; clarify expectations of availability; and take responsibility for having guidelines in flexible work for the employees.

Another key area was to develop shared visions, guidelines, and support for flexible work (34 suggestions), identified by almost all groups (7/8). This key area consisted of three sub areas: clarify guidelines for flexible work and increase information about them, increase support for employees in how to adopt flexible work, and work for a shared vision regarding flexibility. Within this key area, participants requested clearer guidelines about how to work flexibly, for example regarding worktime, physical presence at the workplace, and expectations of availability. In addition, it was frequently mentioned that the guidelines needed to be used throughout the whole organization to create standards of flexible work and potential working methods. It was also suggested to develop a permissive culture with an increased understanding and respect for colleagues' use of flexibility. 


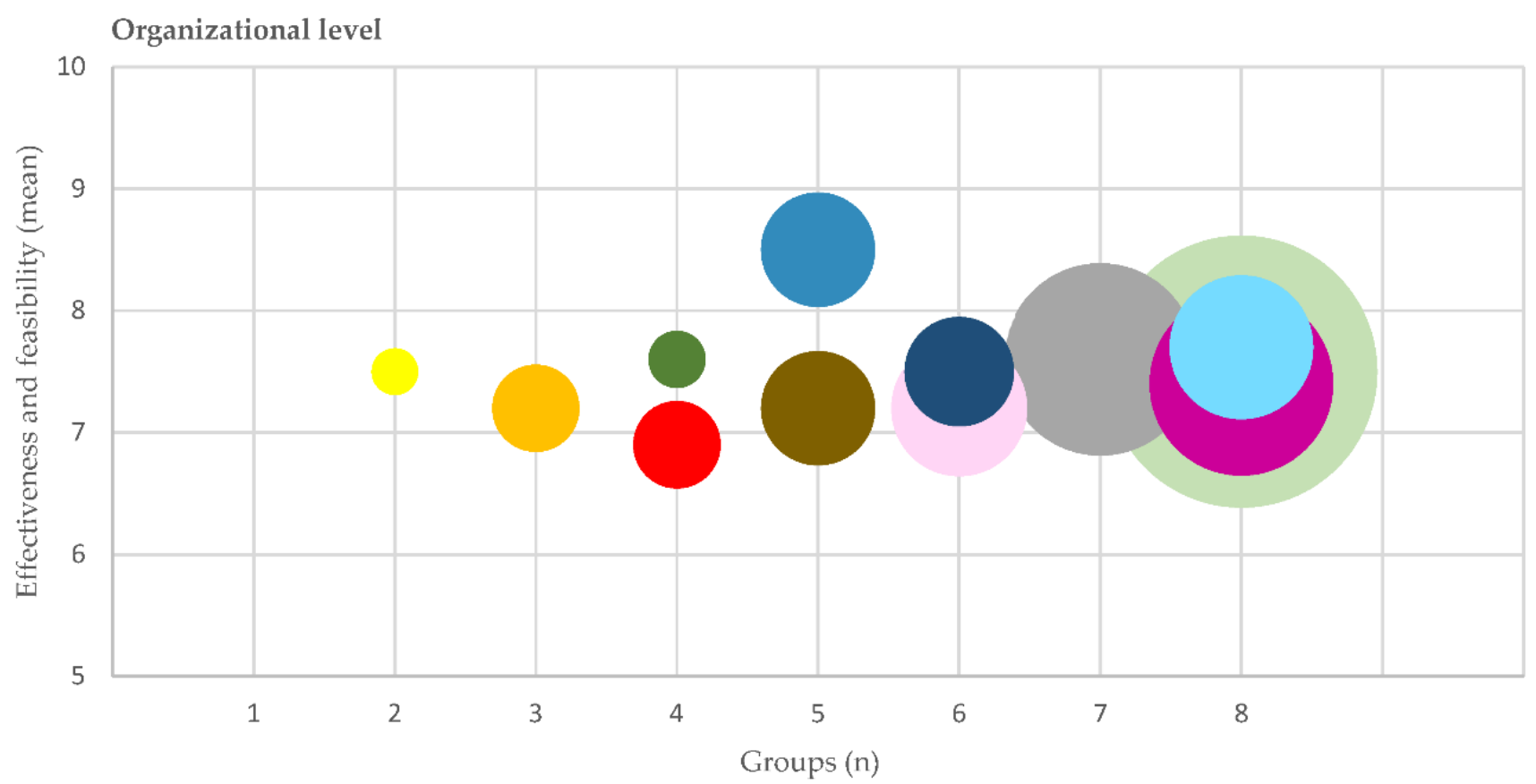

- Leadership in flexible work

- Develop shared visions, guidelines and support for flexible work

- Development of technical systems that enable efficient flexible work

- Increase knowledge about flexible work

Organization of work to reduce job demands and increase recovery

- Improvements of the physical working environment

- Organization of work to increase effectiveness

- Improvements of the work process in activity based offices

- Encourage wellness and physical activity during working hours

- Clarify the support of central functions and role descriptions

- Improvements of social community in flexible work

- Create better conditions for teleworking

Strengthen the culture for respectful behavior in flexible work

Figure 3. Key areas for improvement at the organizational level. Bubble size is proportional to the number of suggestions mapped to each area. The x-axis indicates the number of departments who contributed suggestions to each key area (possible range 0-8); the $y$-axis indicates the mean of all effectiveness and feasibility scores (scale 0-10) for all suggestions in each key area. Note, values for two of the key areas (improvement of the physical working environment and organization of work to reduce job demands and increase recovery) were so similar that the bubbles overlap, and the bubble for improvements of the physical working environment is not visible in the diagram. The only difference between these two was for effectiveness and feasibility mean score (7.5-improvement of the physical working environment and 7.2—organization of work to reduce job demands and increase recovery).

The third key area within the organizational level was development of technical systems that enable efficient flexible work (29 suggestions), identified by all eight groups. This key area was divided into three sub areas; improve technology and simplify technical systems for more efficient work, increase support for digital flexible work, and customize technical equipment based on individual needs. The most stated suggestions within this key area concerned increased technical conditions and support to facilitate flexible work, for example, by providing good technical solutions for teleworking, and to increase knowledge about how to use and customize the technical solutions to suit individual needs. Other examples of key areas within the organizational level were increased knowledge about 
flexible work, organization of work to reduce job demands, increase effectiveness and recovery, and improvements in the physical work environment. The highest mean value for combined effectiveness and feasibility scores was found for the key area encourage wellness and physical activity during working hours (mean $=8.5$ ). This key area was mentioned by five groups and included 12 suggestions. Effectiveness and feasibility mean scores for the other key areas ranged from 6.9 to 7.7 .

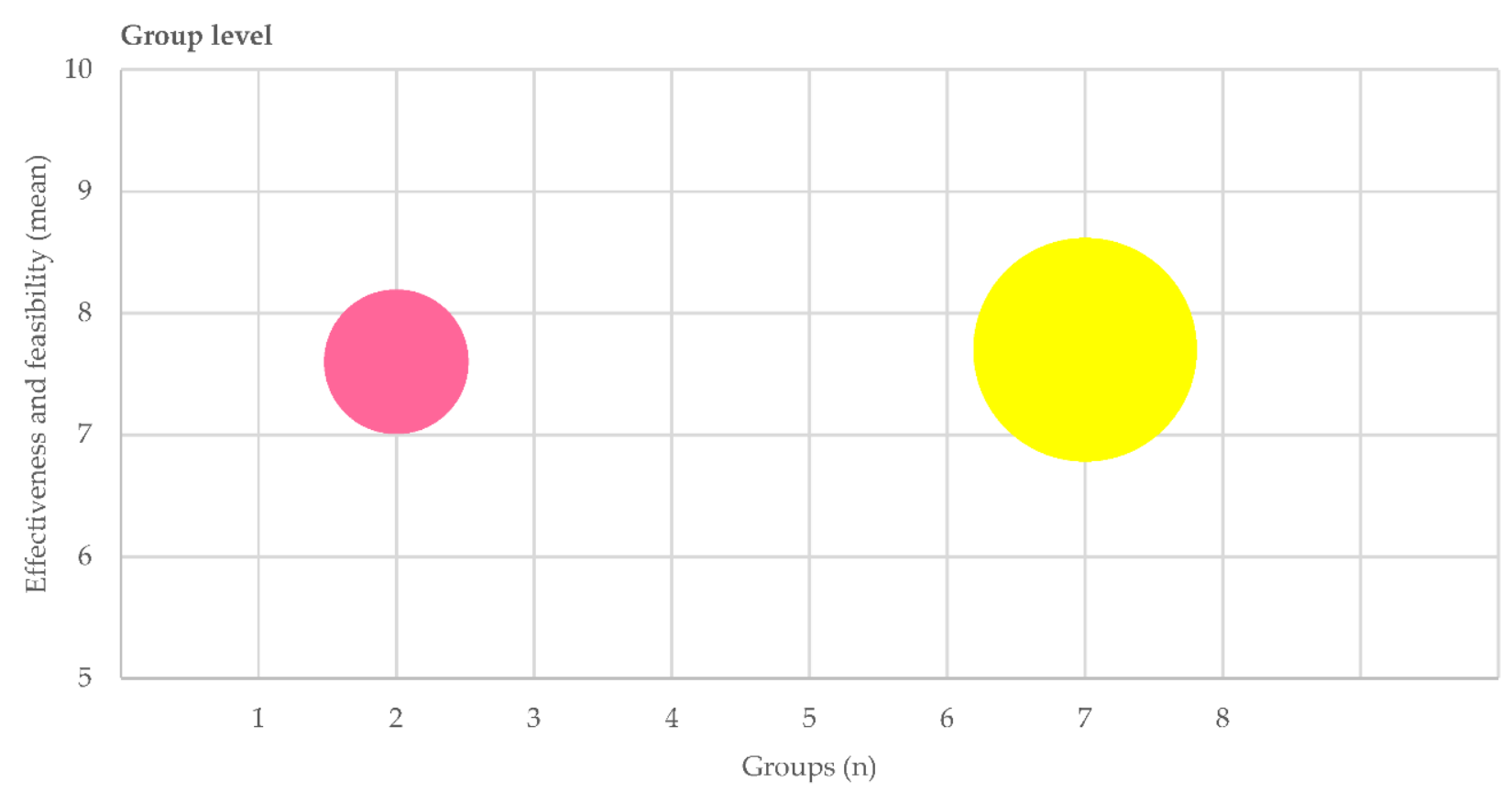

Figure 4. Key areas for improvement at the group level. Bubble size is proportional to the number of suggestions mapped to each area. The $x$-axis indicates the number of departments who contributed suggestions to each key area (possible range 0-8); the $y$-axis indicates the mean of all effectiveness and feasibility scores (scale 0-10) for all suggestions in each key area.

\subsection{Group Level}

At the work group level, the key area comprising the highest number of suggestions, and contributions from seven groups was creating common rules regarding availability in flexible work (12 suggestions), with suggestions from seven of eight groups (see Figure 4). In this key area, participants requested increased communication and common rules within the work group regarding working hours, physical presence at the office, and expectations of availability during free time. The key area with the second largest number of suggestions (5) from two groups was, developing common office rules for activity-based working, for example, how to behave to not disturb colleagues' work. Effectiveness and feasibility mean scores almost equally for the two key areas (range 7.6 to 7.7.).

\subsection{Individual Level}

At the individual level, the key area comprising the highest number of suggestions, and contributions from seven groups was clarification of own availability (15 suggestions) (see Figure 5). This key area included two sub areas; clarify own availability and clarify own work location during the day. The employees suggested technical tools such as Outlook and Skype could be used to communicate availability, work location and when colleagues could expect responses. The concrete suggestions also aimed to increase control over availability by turning off the phone during weekends and vacations, and respecting colleagues posted work hours. The second most frequently mentioned suggestions were grouped to the key area prioritizing breaks to promote recovery, which was based on four suggestions from three of the eight groups. In this key area, the participants emphasized the importance of scheduling lunch and breaks in the calendar as a reminder to have opportunities for recovery during working hours, especially when working from home. The third key area, 
personal responsibility for health and work environment included two suggestions from a single group concerning individuals' own responsibilities to improve health and the work environment by making suggestions for improvements to the organization. Effectiveness and feasibility mean scores almost equally for the three key areas (range 7.2 to 8.3).

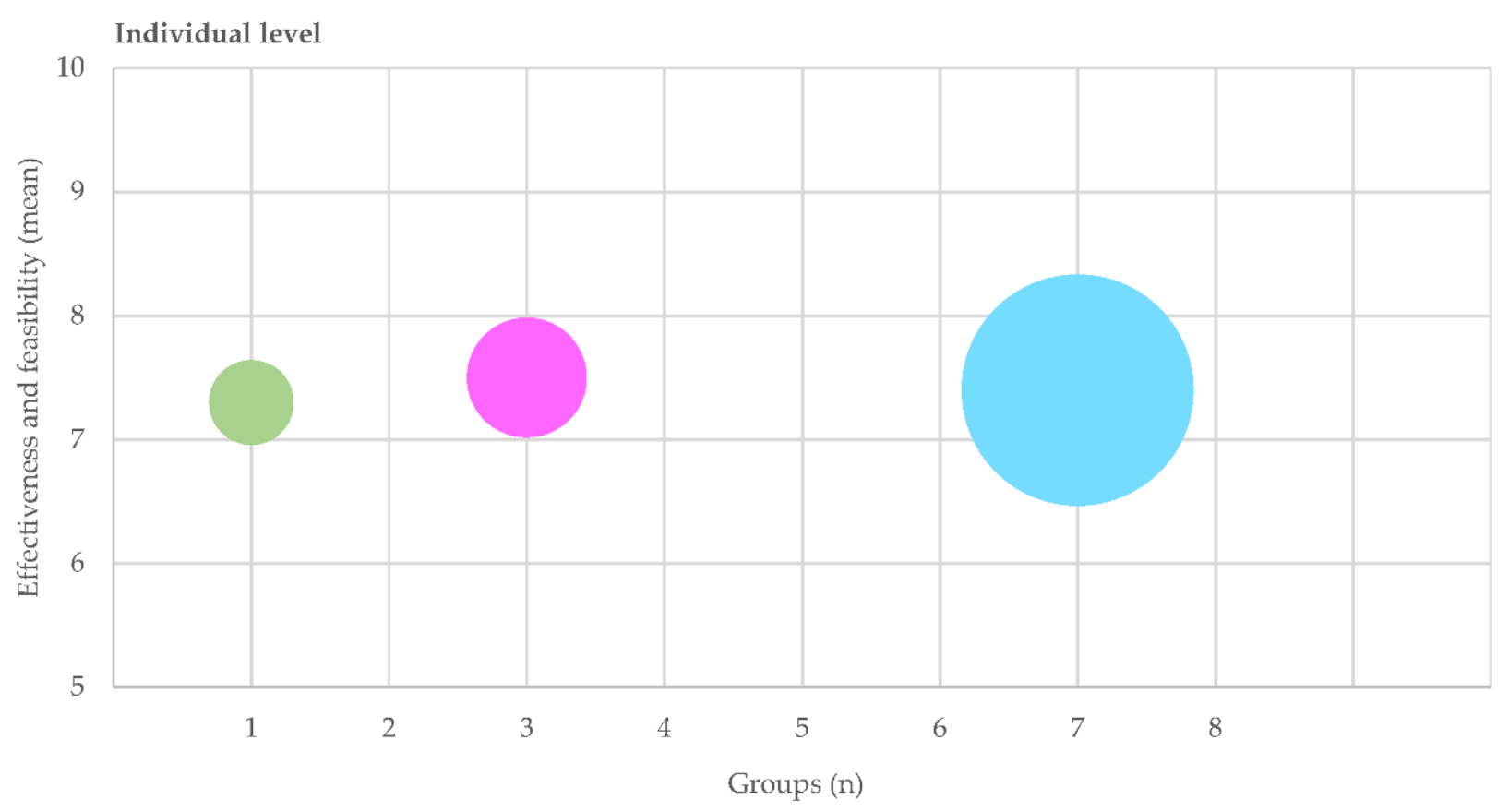

- Clarification of availability in flexible work

- Prioritize breaks to promote recovery

- Personal responsibility for health and work environment

Figure 5. Key areas for improvement at the individual level. Bubble size is proportional to the number of suggestions mapped to each area. The x-axis indicates the number of departments who contributed suggestions to each key area (possible range 0-8); the $y$-axis indicates the mean of all effectiveness and feasibility scores (scale 0-10) for all suggestions in each key area.

\section{Discussion}

In this study, we used a participative approach to collect employee suggestions to promote good work environment and health in flexible work, categorized them by O-G-I level (organizational, work group and individual), and then identified key areas for the company to target. The eight focus groups resulted in 279 concrete suggestions, most of which comprised organizational level initiatives $(86 \%)$, but also included group $(6 \%)$ and individual $(8 \%)$ level changes. The key areas comprising the highest number of suggestions, and contributions from seven or more of the eight groups were leadership in flexible work, develop shared visions, guidelines, and support for flexible work and development of technical systems that enable efficient flexible work.

Leadership in flexible work (organizational level) comprised the highest number of concrete suggestions, and contributions from all groups. Particularly, employees requested a structure-oriented leadership behavior that would clarify boundaries of flexible work, such as when and where to work, expectations of availability and acceptable response times to colleague questions. Previously, relation-oriented leadership behavior has been used as a basis for a successful manager, in interaction with structure- and change oriented behavior [19]. However, flexible work arrangements may cause other problems for employees than traditional work, which may require different types of leadership behaviors. For example, organization of work has presented as a leadership problem when the manager and employees do not meet physically [20]. This may explain why a structural leadership behavior becomes particularly important in flexible work. 
In telework, communication is a known major problem from a leadership perspective $[20,21]$. Clear communication may fulfill employees request to clarify when and where to work, expectations of availability and acceptable response times to colleague questions. Thus, it is important to adapt the leadership to a more flexible way of working [20]. However, more research addressing successful leadership behavior in flexible work arrangements is needed.

On an organizational level, employees also required conditions to work more efficiently in flexible work, for example by adapting technical systems based on the need of the employees and by education and common guidelines to improve effectiveness of meetings, emails and communication. Overall, common guidelines and a shared vision concerning flexible work in the organization was required by the focus groups. It is common that formal policies are not communicated or that employees do not receive support in transferring the policy into practice [22]. A supportive culture within the organization is especially important to reduce the gap between formal policy and practice [22]. We also found a need for common guidelines on how to apply flexible work (e.g., regarding availability), which was evident by the numerous suggestions for improvement at all O-G-I levels. At an individual level, this was reflected by the key area with most concreate suggestions concerned individuals' responsibilities to use technical tools to clearly communicate their availability and location. This suggests that organizations should focus on adapting working conditions to flexible work arrangements to promote health and well-being. These adjustments apply both to a shared vision in the organization, leadership behavior, common rules within the work group and develop technical conditions (ICT) to work flexibly.

In summary, $86 \%$ of the concrete suggestions for improvement implicated organizational level changes (i.e., the organization is responsible for the implementation of the suggestions, but the interventions can be directed to both groups- and individuals, e.g., education about flexible work). Even though our results suggest the bulk of the responsibility is on the organization to improve working conditions in flexible work, employees also suggested changes to be made at group and individual levels. This agrees with previous research identifying risk factors for employees' work-life balance at an organizational level (unstructured and informal policies), group level (expectations of availability and to work more than agreed), and individual level (e.g., overtime work and weak boundary management) [16]. However, it is possible that fewer suggestions targeted group and individual levels because people tend to prefer solutions that do not require them to change their own behaviors [23]. Estimates of mean effectiveness and feasibility were generally high and only marginal differences were found between the O-G-I levels. This indicates that changes at all O-G-I levels are perceived as feasible and effective for promoting a good working environment and health in flexible work arrangements among the employees. Accordingly, interviews with managers may have led to different results.

\subsection{Strengths and Limitations}

This study used a participative approach to generate concrete suggestions which were mapped into key areas requiring action for a sustainable work environment and improved health in flexible work arrangements. Previous research [14] found positive effects on sustainable behavioral change by using a participative approach, and according to our knowledge, this is the first study using a participatory approach in the concept of flexible work arrangements. Another strength is the use of Tree diagram to systematically identify concrete suggestions of improvement for a sustainable and health promoting work environment and estimate their effectiveness and feasibility in the organization. The mapping of suggestions in O-G-I levels may be crucial for the development of successful interventions [12].

Focus group interviews allowed us to systematically collect opinions from multiple participants and departments to ensure organization-wide representation. Our results may have limited generalizability due to different needs in different organizations. However, 
flexible work arrangements are common worldwide and the process of identifying concrete suggestions for improvement may be applicable in other organizations.

COVID-19 has forced employees to work even more flexibly, especially when it comes to work from home [3]. Our study was conducted before the pandemic and key areas in the organization may have changed since then. However, our findings agree well with recent research during the pandemic. For example, development of organizational policy for work flexibility was recently proposed as a key factor for adapting work to COVID-19 related changes [24]. Strengthening organizational culture, increasing the technical feasibility of telework, and clarifying availability [25] have also been recommended along with adapting leadership to new ways of working [3]. Thus, it is likely that the identified key areas for improvement in this study are still important for creating sustainable and health promoting work during and beyond the pandemic.

The interview groups were divided by departments, which may have facilitated confidence and an open discussion as the participants already knew each other. On the other hand, the familiarity could have prevented participants from disagreeing, for example, if a suggestion concerned an improvement within the group. Thus, group dynamics may have affected participants' comfort in expressing opinions. In addition, in group interviews, informal leaders can dominate the discussion, therefore biasing the data. The moderator attempted to avoid dominating personalities by involving all participants in the discussions, and overall, the researchers experienced a dynamic atmosphere in the groups. Many of the key areas were identified by several groups, which increases the generalizability within the organization. The study included only 45 of the 4900 employees with flexible work arrangements within the organization. The inclusion or more employees and/or managers could have led to different concrete suggestions and subsequently key areas.

More research is required to investigate how organizations with flexible work arrangements can contribute to sustainable and health promoting work. Intervention studies are needed to identify effective and sustainable interventions with respect to flexible work, health, and performance.

\subsection{Practical Implications}

Our results emphasize 18 key areas of improvement at three levels within the organization (organizational, work group and individual). Our results, if implemented and evaluated, may lead to evidence-based recommendations and guidelines for organizations with flexible work, and therefore contribute to further investigations and evaluations of interventions within similar populations. More specifically, the results of this study can be useful to develop and implement interventions targeting different levels of the organization. At the organizational level, the company may focus on supporting managers in developing new competences relevant to managing flexible workers and developing a shared vision of flexible work and work guidelines. Organizations may need to help employees find strategies at the individual and group levels for organizing work to reduce job demands and improve effectiveness in flexible work, for example through seminars addressed to improve communication and clarify rules for effective meetings and availability within the work group. It is also important that work groups take initiatives to discuss definitions and guidelines for flexible work. Our results also suggest individuals clearly communicate working hours, locations and expected response times; this can be carried out using technical tools or systems such as, Outlook, Skype, or Teams. The individual may need to use their work calendar to schedule breaks, time for lunch and workday hours and learn to follow their schedule to ensure opportunities for recovery. It may be beneficial to implement several interventions at the same time, but at different levels since interventions at one level may interact with and facilitate changes on other levels [12]. For example, if an intervention is addressed to increase employees' work-life balance by clarifying availability, a common approach within the whole work group can be important. Otherwise, it may be difficult for the individual to maintain the new way of working if the colleagues do not work in the same way or do not respect your new working methods. 


\section{Conclusions}

The aim of this study was to use a participatory approach to identify concrete suggestions and key areas for improvement that were considered relevant, effective, and feasible for promoting good work environment and health at organizational, work group and individual level (O-G-I), among office employees with flexible work arrangements. The suggestions were categorized into 18 key areas, most of which involved organizational level change, but also included suggestions for group and individual level initiatives. The key areas comprising the highest number of suggestions, and contributions from seven or all eight groups were leadership in flexible work and the need to develop shared visions and guidelines, and more support for the employees. Creating common rules regarding availability and activity-based working was a key area at the group level and taking responsibility to communicate availability and prioritizing breaks to promote recovery was a key at the individual level. In conclusion, the participatory process was effective in obtaining concrete suggestions and key areas in need of improvement, which may provide an action plan that can guide organizations in developing interventions to promote good work environment and health in flexible work.

Author Contributions: Conceptualization, methodology, data processing and analysis, S.B., D.M.H., C.Z., J.L., J.E. and H.J.; writing-original draft preparation, S.B.; writing—review and editing, D.M.H., C.Z., J.L., J.E. and H.J.; project administration, H.J., J.L. and C.Z.; funding acquisition, H.J. All authors have read and agreed to the published version of the manuscript.

Funding: The Swedish Transport Administration (Dnr. 2016/425/1) and the Swedish research Council for Health, Working life and Welfare (Forte Dnr. 2009-1761), founded the study. The funders had no influence on the collection, processing, analysis or reporting of data.

Institutional Review Board Statement: The study was conducted according to the guidelines of the Declaration of Helsinki, and approved by the Institutional Review Board of Regional Ethical Board in Uppsala (2016/452/1).

Informed Consent Statement: Informed consent was obtained from all subjects involved in the study.

Data Availability Statement: Supporting data are available by reasonable request (S.B. email: sofie.bjarntoft@hig.se).

Acknowledgments: The authors would particularly like to thank the Swedish Transport Administration for their collaboration in the study. We also want to thank Kristina Palm, Hans Richter, and Jennie Jackson for valuable feedback on the manuscript.

Conflicts of Interest: The authors declare no conflict of interest.

\section{References}

1. Eurostat. Flexibility at Work-Statistics. Available online: https://ec.europa.eu/eurostat/statisticsexplained/index.php?title= Flexibility_at_work_statistics\#Employers_and_clients_mainly_decide_on_working_time_in_Europe (accessed on 27 August 2021).

2. The European Commission's Science and Knowledge Service. Telework in the EU before and after the COVID-19: Where We Were, Where We Head to. Science for Policy Briefs. 2020. Available online: https:/ / ec.europa.eu/jrc/sites/jrcsh/files/jrc120945_ policy_brief_-_COVID_and_telework_final.pdf (accessed on 27 August 2021).

3. Contreras, F.; Baykal, E.; Abid, G. E-Leadership and Teleworking in Times of COVID-19 and Beyond: What We Know and Where Do We Go. Front. Psychol. 2020, 11, 590271. [CrossRef]

4. Johnson, A.; Dey, S.; Nguyen, H.; Groth, M.; Joyce, S.; Tan, L.; Glozier, N.; Harvey, S.B. A review and agenda for examining how technology-driven changes at work will impact workplace mental health and employee well-being. Aust. J. Manag. 2020, 45, 402-424. [CrossRef]

5. Shifrin, N.V.; Michel, J.S. Flexible work arrangements and employee health: A meta-analytic review. Work Stress 2021. [CrossRef]

6. Kumar, S.; Sarkar, S.; Chahar, B. A systematic review of work-life integration and role of flexible work arrangements. Int. J. Organ. Anal. 2021. [CrossRef]

7. Beigi, M.; Shirmohammadi, M.; Stewart, J. Flexible Work Arrangements and Work-Family Conflict: A Metasynthesis of Qualitative Studies among Academics. Hum. Resour. Dev. Rev. 2018, 17, 314-336. [CrossRef]

8. Mellner, C. After-hours availability expectations, work-related smartphone use during leisure, and psychological detachment: The moderating role of boundary control. Int. J. Workplace Health Manag. 2015, 9, 146-164. [CrossRef] 
9. Borgmann, L.S.; Rattay, P.; Lampert, T. Health-related consequences of work-family conflict from a European perspective: Results of a scoping review. Front. Public Health 2019, 7, 189. [CrossRef] [PubMed]

10. Holtermann, A.; Mathiassen, S.E.; Straker, L. Promoting health and physical capacity during productive work: The Goldilocks Principle. Scand. J. Work. Environ. Health 2018, 45, 90-97. [CrossRef] [PubMed]

11. Fox, K.E.; Johnson, S.T.; Berkman, L.F.; Sianoja, M.; Soh, Y.; Kubzansky, L.D.; Kelly, E.L. Organisational- and group-level workplace interventions and their effect on multiple domains of worker well-being: A systematic review. Work Stress 2021, 1-30. [CrossRef]

12. Dellve, L.; Eriksson, A. Health-Promoting Managerial Work: A Theoretical Framework for a Leadership Program that Supports Knowledge and Capability to Craft Sustainable Work Practices in Daily Practice and During Organizational Change. Societies 2017, 7, 12. [CrossRef]

13. Kossek, E.E.; Lewis, S.; Hammer, L.B. Work-life initiatives and organizational change: Overcoming mixed messages to move from the margin to the mainstream. Hum. Relat. 2010, 63, 3-19. [CrossRef] [PubMed]

14. Van Eerd, D.; Cole, D.; Irvin, E.; Mahood, Q.; Keown, K.; Theberge, N.; Village, J.; St Vincent, M.; Cullen, K. Process and implementation of participatory ergonomic interventions: A systematic review. Ergonomics 2010, 53, 1153-1166. [CrossRef] [PubMed]

15. Wilson, J.R. Ergonomics and participation. In Evaulation of Human Work, 2nd ed.; Wilson, J.R., Corlett, E.N., Eds.; CRC-Press: Boca Raton, FL, USA, 1995.

16. Bjärntoft, S.; Hallman, D.; Mathiassen, S.E.; Larsson, J.; Jahncke, H. Occupational and Individual Determinants of Work-life Balance among Office Workers with Flexible Work Arrangements. Int. J. Environ. Res. Public Health 2020, 17, 1418. [CrossRef] [PubMed]

17. Klefsjö, B.; Eliasson, L.; Kennerfalk, L.; Lundbäck, A.S.M. De SJu Ledningsverktygen. För Effektivare Planering av Förbättringsarbete; Studentlitteratur: Lund, Sweden, 1999.

18. Mizuno, S. Management for Quality Improvement: The Seven New QC Tools; Productivity Press: Cambridge, UK, 1998.

19. Larsson, J.; Vinberg, S. Leadership behaviour in successful organisations: Universal or situation-dependent? Total Qual. Manag. Bus. Excell. 2010, 21, 317-334. [CrossRef]

20. Kirchner, K.; Ipsen, C.; Hansen, J.P. COVID-19 leadership challenges in knowledge work. Knowl. Manag. Res. Pract. 2021. [CrossRef]

21. Wojcak, E.; Bajzikova, L.; Sajgalikova, H.; Polakova, M. How to Achieve Sustainable Efficiency with Teleworkers: Leadership Model in Telework. Proc.-Soc. Behav. Sci. 2016, 229, 33-41. [CrossRef]

22. Brough, P.; O'Driscoll, M.P. Organizational interventions for balancing work and home demands: An overview. Work Stress 2010, 24, 280-297. [CrossRef]

23. Prochaska, J.O.; Velicer, W.F. The transtheoretical model of health behavior change. Am. J. Health Promot. 1997, 12, 38-48. [CrossRef] [PubMed]

24. De Lucas Ancillo, A.; Del Val Núñez, M.T.; Gavrila, S.G. Workplace change within the COVID-19 context: A grounded theory approach. Econ. Res. Ekon. Istraz. 2021, 34, 2297-2316. [CrossRef]

25. Fana, M.; Milasi, S.; Napierala, J.; Fernandez-Macias, E.; Gonzalez Vazquez, I. Telework, Work Organisation and Job Quality during the COVID-19 Crisis: A Qualitative Study. Available online: https://www.econstor.eu/handle/10419/231343 (accessed on 27 August 2021). 